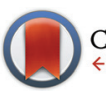

CrossMark

Cite this: Polym. Chem., 2016, 7, 2092

Received 28th January 2016, Accepted 19th February 2016

DOI: 10.1039/c6py00161k

www.rsc.org/polymers

\title{
Facile synthesis of bowl-shaped nitrogen-doped carbon hollow particles templated by block copolymer "kippah vesicles" for high performance supercapacitors $\uparrow$
}

\author{
Zhixing Lin, ${ }^{a}$ Hao Tian, ${ }^{a}$ Fugui $\mathrm{Xu},{ }^{a}$ Xiangwen Yang, ${ }^{a}$ Yiyong Mai ${ }^{\star a}$ and \\ Xinliang Feng ${ }^{a, b}$
}

\begin{abstract}
This paper reports a simple self-assembly strategy towards bowl-shaped carbon-containing hollow particles, as well as an unprecedented potential application for block copolymer vesicles in energy storage. Kippah vesicles (fully collapsed vesicles), formed by solution self-assembly of an amphiphilic polystyreneblock-poly(ethylene oxide) block copolymer, were employed as the template to guide the formation of bowl-shaped nitrogen-doped carbon hollow particles (BNCHPs). As electrode materials of supercapacitors, BNCHPs exhibit superior electrochemical performance. In particular, compared with their spherical counterpart, BNCHPs largely increase their volumetric packing density, leading to much higher volumetric capacitance or volume reduction of electrodes, which is desired for practical supercapacitor devices.
\end{abstract}

\section{Introduction}

The self-assembly of block copolymers (BCPs) has attracted much attention in recent decades because it provides a versatile approach towards diverse supramolecular assemblies of controllable morphology, including spheres, cylinders, and vesicles, among others. ${ }^{1,2}$ These assemblies show great potential applications in a wide range of fields, such as biomedicine, electronics, catalysts, energy storage and conversion, etc. ${ }^{1,2}$ As one specific example at a more advanced level among the various assemblies, BCP vesicles (or polymersomes) are of considerable interest due to their hollow sphere structure and good stability. ${ }^{3-9}$ The current potential applications of BCP vesicles, e.g. in drug or gene delivery, mostly rely on the presence of the void cavities which offer a possibility for the encapsulation of various hydrophilic substances. ${ }^{1-9}$ However, although with a wide range of promising uses, BCP vesicles have rarely been explored for energy storage purposes. In the present study, we developed an unprecedented potential application for BCP vesicles in energy storage, by taking advantage of their deformation to eliminate the cavities.

${ }^{a}$ School of Chemistry and Chemical Engineering, Shanghai Jiao Tong University, 800 Dongchuan Rd, Shanghai 200240, P. R. China.E-mail: mai@sjtu.edu.cn ${ }^{b}$ Department of Chemistry and Food Chemistry, Technische Universität Dresden, Mommsenstrasse 4, 01062 Dresden, Germany

$\dagger$ Electronic supplementary information (ESI) available: ESI figures and tables. See DOI: $10.1039 / \mathrm{c} 6$ py00161k
"Kippah vesicles", ${ }^{10}$ i.e. fully collapsed vesicles, were prepared in this study by the self-assembly of an amphiphilic polystyrene-block-poly(ethylene oxide) (PS- $b$-PEO) block copolymer in solution (Fig. 1). Through employing the kippah vesicles as the template to support the polymerization of dopamine, followed by the pyrolysis of the resulting polydopamine@kippah-vesicle nanobowls at $900{ }^{\circ} \mathrm{C}$, bowl-shaped nitrogen-doped carbon hollow particles (BNCHPs) were suc-

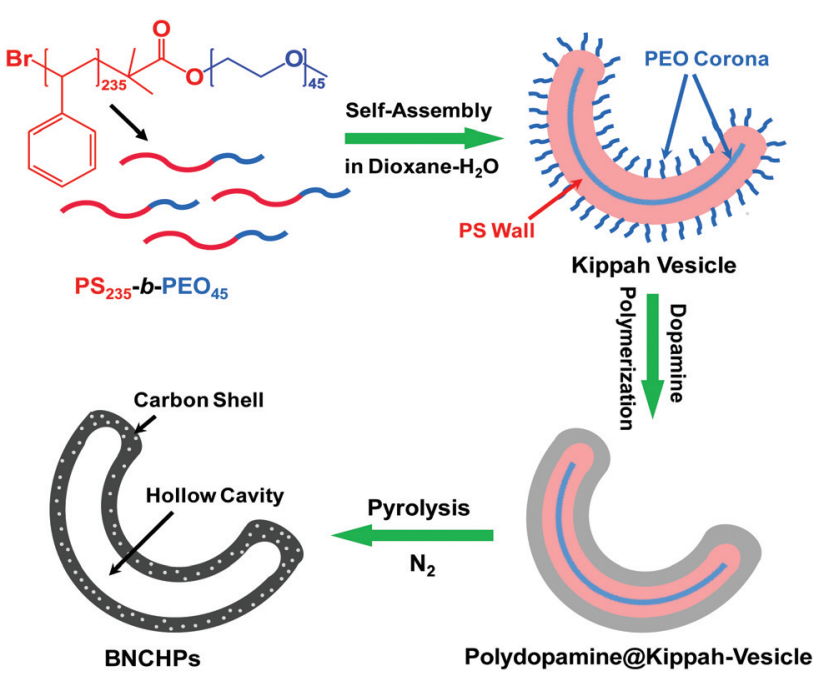

Fig. 1 Schematic illustration of the preparation of BNCHPs using kippah vesicles as the templates. 


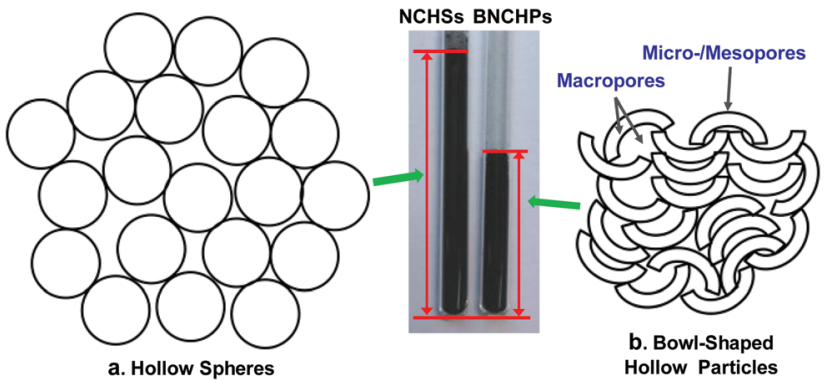

Fig. 2 A schematic illustration showing the comparison of the packing volumes of hollow spheres and bowl-like hollow particles of the same weights. The diameter and the number of the drawn hollow spheres (a) are the same as those of the bowl-like hollow particles (b). The photo in the middle presents the real products of NCHSs (left) and BNCHPs (right) of the same weights in glass tubes. Apparently, the packing density of BNCHPs is much larger than that of NCHSs.

cessfully synthesized. The BNCHPs possess an average diameter of $450 \pm 55 \mathrm{~nm}$ and an average carbon shell thickness of $23 \pm 1 \mathrm{~nm}$, associated with a large specific surface area of $370 \mathrm{~m}^{2} \mathrm{~g}^{-1}$ and a high nitrogen content of $\sim 4 \mathrm{wt} \%$. Such bowlshaped hollow particles hold promise as electrode materials for energy storage devices such as electrochemical supercapacitors. Compared with their spherical counterpart, i.e. hollow carbon-containing spheres that have been one of the appealing electrode materials for supercapacitors, ${ }^{11-16}$ the bowl-shaped hollow particles largely increase their packing density because of the much smaller empty cavities (Fig. 2), which may result in higher volumetric capacitance or volume reduction of electrodes. This merit is desired for practical supercapacitor devices. Remarkably, acting as electrode materials in supercapacitors, BNCHPs exhibit high specific capacitance (e.g. 385 $\mathrm{F} \mathrm{g}^{-1}$ at $0.1 \mathrm{~A} \mathrm{~g}^{-1}$ ), high volumetric capacitance (e.g. 410 $\mathrm{F} \mathrm{cm}^{-3}$ at $0.1 \mathrm{~A} \mathrm{~g}^{-1}$ ), good capacitance retention, and outstanding cycling stability with no capacitance loss after 10000 cycles. Such excellent electrochemical performance is superior to those of their spherical counterpart, namely $\mathrm{N}$-doped carbon hollow spheres (NCHSs), as well as many reported carbon materials, such as $\mathrm{N}$-doped carbons and graphenebased materials. ${ }^{17-26}$

\section{Experimental section}

\section{Materials}

The $\mathrm{PS}_{235}-b-\mathrm{PEO}_{45}$ block copolymer was prepared in a previous study. ${ }^{27}$ Monomethoxy poly(ethylene oxide) (monomethoxyPEO2000), 2-bromoisobutyryl, and styrene were purchased from Aldrich Corp. Dopamine hydrochloride, 1,4-dioxane, tris (hydroxymethyl)aminomethane (Tris), and tetrahydrofuran (THF) were purchased from Adamas Corp. Polystyrene (PS) hollow spheres were purchased from Dow Company. All other chemicals were purchased from Aladdin Reagent (Shanghai) and used without further purification. Organic solvents were distilled before use. Deionized water was used in all experiments.

\section{Self-assembly of $\mathrm{PS}_{235}-b-\mathrm{PEO}_{45}$ block copolymer into kippah vesicles in solution}

In a typical procedure, $\mathrm{PS}_{235}-b-\mathrm{PEO}_{45}(10 \mathrm{mg})$ was dissolved in 1,4-dioxane $(1 \mathrm{~mL})$ in a $5 \mathrm{~mL}$ capped vial with a magnetic stirrer. The solution was stirred for one hour at room temperature. Then, $1 \mathrm{~mL}$ MilliQ water was added to the organic solution at a rate of $0.25 \mathrm{~mL} \mathrm{~h}^{-1}$ under stirring. Afterwards, $20 \mathrm{~mL}$ MilliQ water was quickly added into the solution to quench the morphology of the formed aggregates.

\section{Synthesis of BNCHPs}

$20 \mathrm{mg}$ dopamine hydrochloride was added to the as-obtained solution of the kippah vesicles, and the mixture was stirred mildly for one hour at room temperature. Then, $18 \mathrm{mg}$ Tris was added into the mixture, followed by stirring for $48 \mathrm{~h}$ at room temperature for the polymerization of the dopamine. The product was collected and purified over 5 cycles of centrifugation and wash with pure water. Finally, the collected product was freeze-dried for 12 hours to yield polydopamine@ kippah-vesicle particles.

The as-prepared polydopamine@kippah-vesicle particles were loaded in a quartz boat and then placed in a tube furnace. The BNCHPs were obtained by the pyrolysis of the particles under nitrogen at $400{ }^{\circ} \mathrm{C}$ for 3 hours and then $900{ }^{\circ} \mathrm{C}$ for another 2 hours. The heating rate was $5{ }^{\circ} \mathrm{C} \mathrm{min}^{-1}$. After the thermal treatment, the resultant products were naturally cooled down to the room temperature.

\section{Synthesis of NCHSs}

The NCHSs were prepared by using PS hollow spheres of an average diameter close to that of the kippah vesicles as the template. The synthesis procedures were similar to those of preparing the BNCHPs, except the use of a different template.

\section{Characterization}

Scanning electron microscopy (SEM) observations were performed on an FEI Sirion-200 (FEI Co., USA) field emission scanning electron microscope. The samples were dispersed in water and sonicated for 15 minutes. SEM samples were prepared by dropping a drop of the water dispersion on silicon wafers, followed by air-drying for 24 hours. Before coating, the silicon substrates were cleaned in a bath of $100 \mathrm{~mL}$ of $80 \%$ $\mathrm{H}_{2} \mathrm{SO}_{4}, 35 \mathrm{~mL}$ of $\mathrm{H}_{2} \mathrm{O}_{2}$ and then $20 \mathrm{~mL}$ of Milli-Q water for 15 minutes at room temperature. The silicon surface was then dried with compressed nitrogen gas.

Transmission electron microscopy (TEM) studies were conducted on a JEOL-2100 (JEOL Ltd, Japan) electron microscope at an operating voltage of $200 \mathrm{kV}$. The samples were dispersed in water and sonicated for 15 minutes. TEM samples were prepared by dropping a drop of the water dispersion on copper grids, followed by air-drying for 24 hours.

Thermogravimetric analysis (TGA) was carried out on a Q5000IR (TA Instruments, USA) thermogravimetric analyzer 
under nitrogen at a heating rate of $10^{\circ} \mathrm{C}^{\mathrm{min}^{-1}}$ over a temperature range of $50-1000{ }^{\circ} \mathrm{C}$.

Elemental analysis (EA) was performed using a Vario ELIII/ Isoprime (Elementar Co., Germany) isotope ratio mass spectrometer.

X-ray photoelectron spectroscopy (XPS) was conducted on an AXIS Ultra DLD system (Kratos Co., Japan) with Al Ko radiation as the X-ray source.

Brunauer-Emmett-Teller (BET) specific surface areas were measured on an Autosorb-iQA3200-4 sorption analyzer (Quantatech Co., USA) based on $\mathrm{N}_{2}$ adsorption and desorption.

Raman spectra were recorded on an Invia/Reflrx Lasser Micro-Raman spectroscope (Renishaw, England) with an excitation laser beam wavelength of $532 \mathrm{~nm}$.

Electrochemical performance evaluation: electrochemical performance of BNCHPs or NCHSs as electrode materials of supercapacitors was evaluated on an EG \& potentiostat/ galvanostat Model 2273 advanced electrochemical system. Cyclic voltammetry (CV) and galvanostatic charge-discharge measurements were performed in a three-electrode system with $1 \mathrm{M} \mathrm{H}_{2} \mathrm{SO}_{4}$ electrolyte. The working electrodes were prepared by mixing $80 \mathrm{wt} \%$ powdered active materials, $10 \mathrm{wt} \%$ carbon black (Mitsubishi Chemicals, Inc.), and $10 \mathrm{wt} \%$ polytetrafluoroethylene (PTFE) binder; Ni foams with a surface area of $\sim 1 \mathrm{~cm}^{2}$ were used as the current collectors. Pt was employed as the counter electrode and an $\mathrm{Ag} / \mathrm{AgCl}$ electrode was used as the reference electrode. The range of electric potential was $0-1 \mathrm{~V}(\mathrm{Ag} / \mathrm{AgCl})$ at different scan rates and different current densities at the ambient temperature. Nyquist plots of the samples were recorded by applying a sine wave with an amplitude of $5.0 \mathrm{mV}$ over a frequency range of $100 \mathrm{kHz}-0.01 \mathrm{~Hz}$.

\section{Results and discussion}

The fabrication procedure of BNCHPs is illustrated in Fig. 1. Briefly, the kippah vesicles were prepared by the addition $\left(0.25 \mathrm{~mL} \mathrm{~h}^{-1}\right)$ of deionized water into a dioxane solution (10 wt\%) of $\mathrm{PS}_{235}-b-\mathrm{PEO}_{45}$ (the number denotes the degree of polymerization of the polymer block); when $50 \mathrm{wt} \%$ water content was reached, the formed assemblies were quenched by rapidly adding a 10-fold amount of water. Upon water addition, spherical vesicles can be formed via the self-assembly of amphiphilic $\mathrm{PS}_{235}-b-\mathrm{PEO}_{45}$ above the critical water content, driven by the hydrophobic interaction of PS blocks. ${ }^{27}$ In the vesicles, the hydrophobic PS blocks construct the vesicle walls and the hydrophilic PEO segments form the corona. With increasing water contents, especially during the rapid addition of water, the dioxane diffuses to the exterior of the vesicles and water penetrates into the interior, driven by the difference of water concentration between the interior and the exterior of the vesicles. Since the diffusion of the dioxane through the hydrophobic PS wall is much faster than that of water, a negative pressure is generated inside the vesicles. Thereby, under optimized experimental conditions by adjusting the rate of addition of water and the water content, the negative pressure is high enough to cause the full collapse of the vesicles, yielding the kippah vesicles. ${ }^{2,10}$ Note that the self-assembly process is remarkably simple, which requires only one step of water addition. Afterwards, dopamine was added into the vesicle solution, which could be absorbed on the PEO coronae via hydrogen bonding at $\mathrm{pH} \sim 8.5$ (adjusted by Tris); the kippah vesicles acted as the template to support the polymerization of the dopamine, yielding bowl-like polydopamine@kippahvesicle particles. Finally, the template was removed during the carbonization process of the polydopamine@kippah-vesicle particles to afford BNCHPs. To our knowledge, this is the first self-assembly approach towards bowl-shaped hollow carboncontaining structures. Although a few recent studies reported the fabrication of bowl-like hollow particles templated by silica or polystyrene nanobowls, the synthesis of the templates required complicated multistep procedures. ${ }^{28-30}$

The morphology of the kippah vesicles was examined by SEM and TEM (Fig. 3a-c). Clearly, the bowl-shaped structure of the kippah vesicles is identified in the EM images. Statistics based on $c a .200$ particles in the SEM images reveal an average diameter of $425 \pm 60 \mathrm{~nm}$ and a narrow size distribution for the kippah vesicles (Fig. S1†). TEM micrograph (Fig. 3c) shows the bowl-shaped cavity in the center of the kippah vesicles and their average wall thickness is measured to be $c a .50 \mathrm{~nm}$, which is approximately twice that $(c a .27 \mathrm{~nm})$ of the spherical vesicles ${ }^{27}$ proving the merging of two layers of walls after the full collapse of the spherical vesicles. It should be mentioned here that the morphology of the kippah vesicles in solution is the same as that in the dry state during the SEM and TEM observations, because after the addition of an excess of water, the PS blocks in the vesicle wall are desolvated and thus become glassy due to the high glass transition temperature $\left(\sim 110^{\circ} \mathrm{C}\right)$ of PS, resulting in the "freeze" of the kippah structure. ${ }^{2,27}$ The frozen structure also provides a "solid" template for the subsequent preparation of BNCHPs.

After the polymerization of dopamine on the PEO coronae of the kippah vesicles, the bowl-shaped structure is well retained (Fig. 3d-f). The average diameter and wall thickness of the polydopamine@kippah-vesicle particles, compared with those of the kippah vesicles, increase to $475 \pm 57 \mathrm{~nm}$ and $100 \pm 2 \mathrm{~nm}$, respectively, due to the coating of a polydopamine layer. TGA results manifested that the polydopamine@kippahvesicle particles underwent a sharp weight loss at $\sim 400{ }^{\circ} \mathrm{C}$ under nitrogen and a carbon yield of $\sim 30 \mathrm{wt} \%$ remained over $900{ }^{\circ} \mathrm{C}$ (Fig. 4). The weight loss at $\sim 400{ }^{\circ} \mathrm{C}$ was attributed to the degradation and carbonization of PS- $b$-PEO copolymer and polydopamine. ${ }^{27}$ Thus, we first treated the polydopamine@ kippah-vesicle particles at $400{ }^{\circ} \mathrm{C}$ for the carbonization of polydopamine along with the degradation of the kippah vesicle template to maintain the bowl-like structure for the resulting carbon particles, and then increased the temperature to $900{ }^{\circ} \mathrm{C}$ for the complete degradation and carbonization of PS- $b$-PEO and polydopamine. After the thermal treatment, SEM and TEM images reveal that the bowl-like structure was 

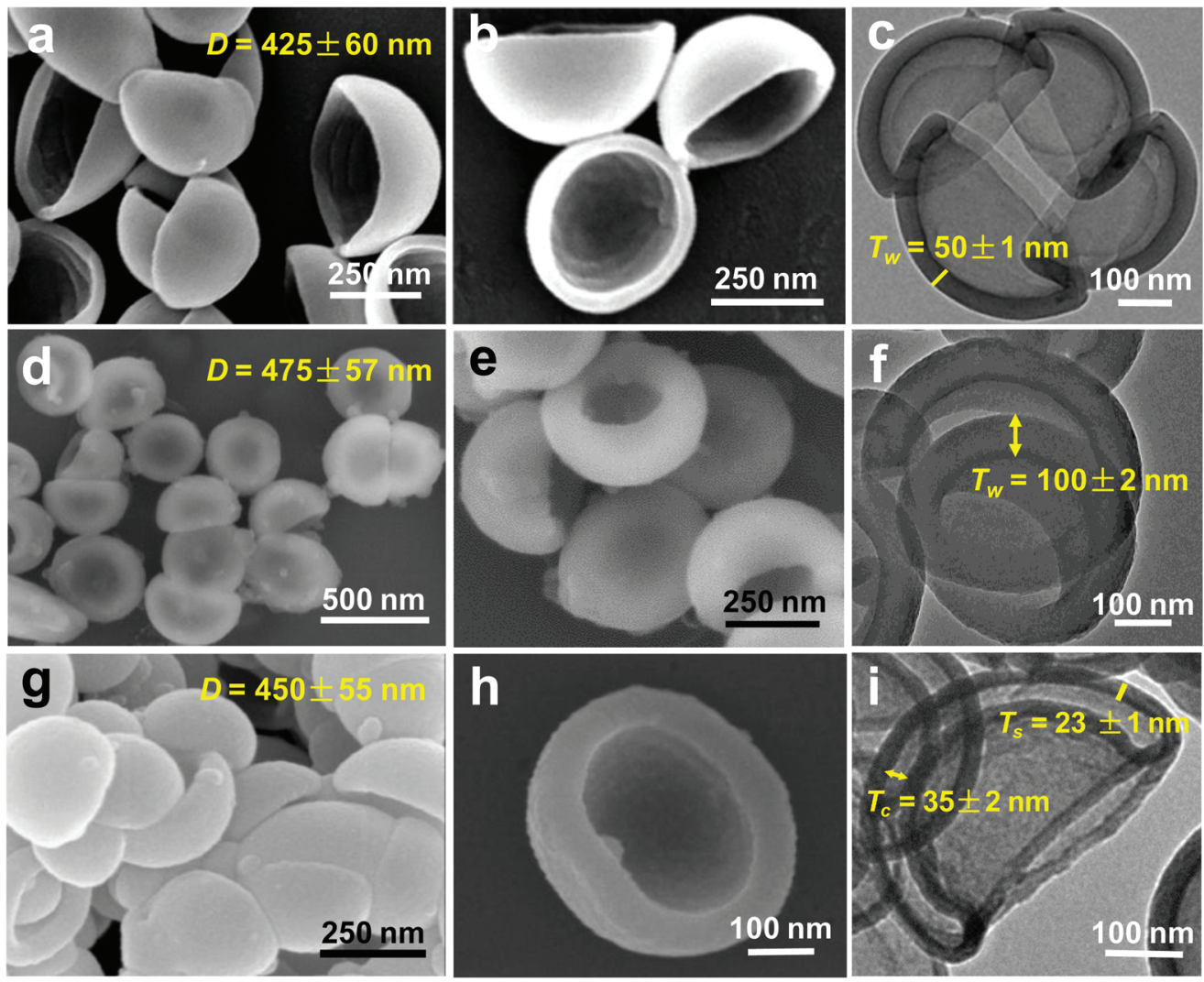

Fig. 3 Typical SEM and TEM micrographs of the kippah vesicles $(a-c)$, polydopamine akippah-vesicle particles (d-f), and BNCHPs ( $g-i)$. In related images, $D$ denotes the average diameters of the particles; $T_{\mathrm{w}}$ expresses the average wall thickness of the kippah vesicles (c) or the polydopamine@ kippah-vesicle particles (f); $T_{\mathrm{s}}$ and $T_{\mathrm{c}}$ in (i) mean the average thicknesses of the carbon shell and the hollow cavity, respectively.

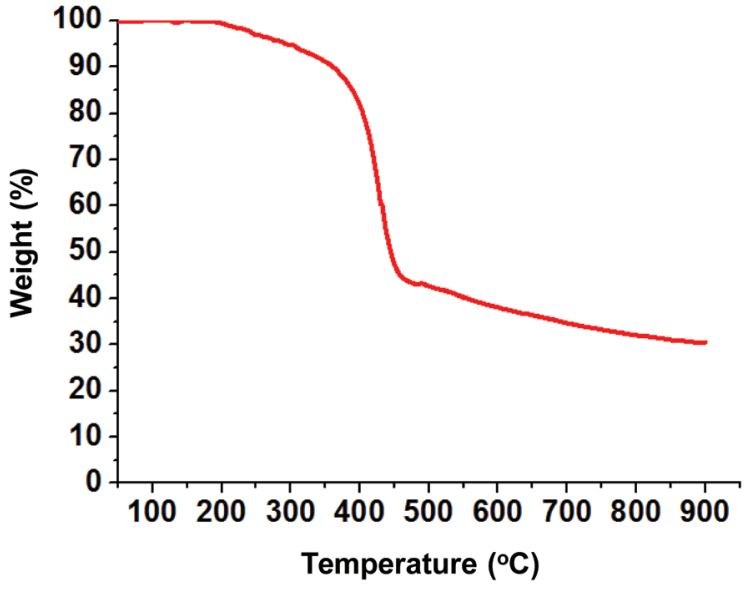

Fig. 4 A typical TGA curve of polydopamine@kippah-vesicle particles. The curve was recorded under a nitrogen atmosphere at a heating rate of $10^{\circ} \mathrm{C} \mathrm{min}^{-1}$.

perfectly retained for the resultant BNCHPs (Fig. 3g-i and $\mathrm{S} 2 \dagger$ ), demonstrating the excellent structural stability. The BNCHPs also possess a narrow size distribution (Fig. S1†) and their average diameter $(450 \pm 55 \mathrm{~nm})$ is close to that of the polydopamine@kippah-vesicle particles. TEM images clearly show the removal of the kippah vesicle template, leaving bowllike hollow space in BNCHPs, which is surrounded by a carbon shell of $c a .23 \pm 1 \mathrm{~nm}$ thickness (Fig. $3 \mathrm{i}$ and $\mathrm{S} 2 \dagger$ ). The average thickness of the hollow cavity in BNCHPs is $35 \pm 2 \mathrm{~nm}$. This value is smaller than the wall thickness $(\sim 50 \mathrm{~nm})$ of the kippah vesicles, owing to the carbonization of PS wall as well as the shrinkage of the cavity during the pyrolysis.

High-resolution TEM (HRTEM) micrographs of the carbon shell reveal the existence of partial graphitic structures and amorphous carbons (Fig. 5a). The Raman spectrum shows two strong peaks at 1345 and $1585 \mathrm{~cm}^{-1}$, which can be assigned to the $\mathrm{D}$ and $\mathrm{G}$ bands of the disordered and graphitized carbons, respectively (Fig. 5b). The higher intensity of the $\mathrm{G}$ band than that of the $\mathrm{D}$ band (intensity ratio of $\mathrm{G}$ to $\mathrm{D} \sim 1.1$ ) suggests a high degree of graphitization for BNCHPs, ${ }^{11}$ confirming the HRTEM result. The graphitization may render BNCHPs good electrical conductivity, which is required for electrode materials of supercapacitors. ${ }^{13}$

The $\mathrm{N}_{2}$ adsorption-desorption isotherm and pore size distribution curves reveal a hierarchically porous structure for BNCHPs (Fig. 5c). The uptake at high relative pressures $\left(P / P_{0}>\right.$ 0.9 ) in the $\mathrm{N}_{2}$ adsorption-desorption isotherm is attributed to the presence of macropores, ${ }^{31}$ which originate from the hollow cavities and the stacking interspace of BNCHPs. The pore size distribution, calculated based on Density Functional Theory 

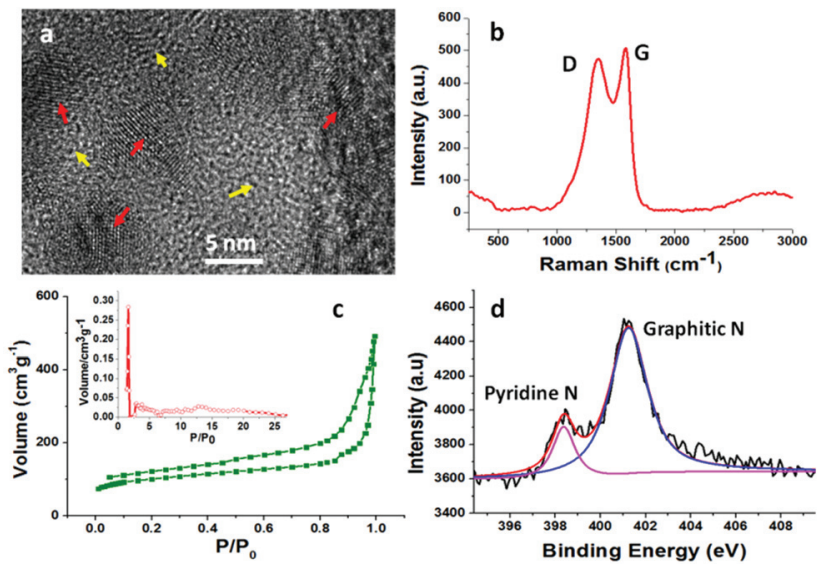

Fig. 5 Microstructure characterization of BNCHPs. (a) A typical HRTEM image of the carbon shell in BNCHPs. It shows the coexistence of amorphous architecture (yellow arrows) and partial graphite crystal structures (red arrows). (b) A typical Raman spectrum of BNCHPs. (c) Nitrogen adsorption-desorption isotherm and pore size distribution (inset). (d) A N1s core-level XPS spectrum.

(DFT), indicates the presence of micropores of $\sim 1.5 \mathrm{~nm}$ in diameter and mesopores of 3-20 nm in BNCHPs. Such a hierarchical porous structure with a large specific surface area of ca. $370 \mathrm{~m}^{2} \mathrm{~g}^{-1}$ is desirable for electrode materials in supercapacitors, as the macropores may act as a reservoir for electrolytes and thus shorten the ion diffusion distances from the exterior to the interior surfaces, while the meso-/micropores in the carbon shells can enhance ion transport and charge storage. $^{19}$

The chemical nature of BNCHPs was studied by elemental analysis and XPS. The elemental analysis shows that the carbon and nitrogen contents in BNCHPs are $84.6 \mathrm{wt} \%$ and $3.7 \mathrm{wt} \%$, respectively. The XPS analysis gives a nitrogen content of $3.1 \mathrm{wt} \%$ for BNCHPs, which is in agreement with the elemental analysis result. In addition, the XPS spectrum manifests the coexistence of graphitic nitrogen $(\sim 401.2 \mathrm{eV})$ and pyridine nitrogen ( 398.3 eV) in BNCHPs (Fig. $5 \mathrm{~d}))^{32}$ The presence of the suitable amount of nitrogen atoms can not only afford pseudocapacitance and high specific surface area, but also enhance the surface wettability with aqueous electrolyte and thus reduce the resistance of electrode materials, compared with undoped porous carbons. ${ }^{13,17}$

The electrochemical performance of BNCHPs as electrode materials was evaluated in a three-electrode system with $1 \mathrm{M}$ $\mathrm{H}_{2} \mathrm{SO}_{4}$ electrolyte. The CV curves of BNCHPs measured at different scan rates exhibit typical double-layer capacitive behavior with high capacitances (Fig. S3a $\dagger$ ). It should be mentioned that some redox peaks are observed in the CV curves, which are probably due to the nitrogen doping that generates pseudocapacitance. $^{22}$ Moreover, the galvanostatic charge/ discharge measurements gave nearly triangular charge/ discharge curves at different current densities (Fig. 6a), indicative of an efficient ion transport throughout the BNCHP electrodes. Based on the discharge curve, the specific capacitance
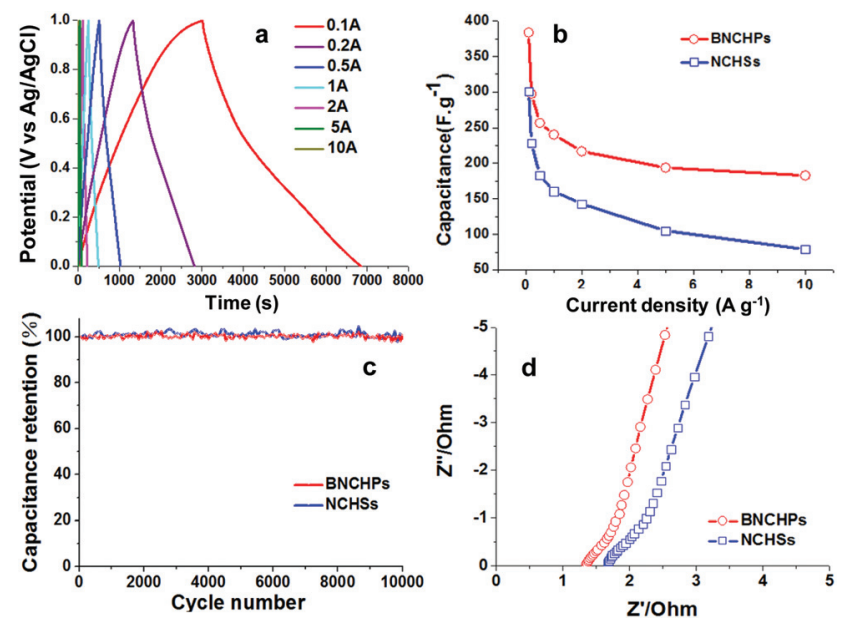

Fig. 6 Electrochemical performance of BNCHPs or NCHSs as electrodes for supercapacitors. (a) Galvanostatic charge/discharge curves of BNCHPs at different current densities in $1 \mathrm{M} \mathrm{H}_{2} \mathrm{SO}_{4}$ electrolyte (the $\mathrm{CV}$ and charge/discharge curves of NCHSs are presented in Fig. S3b and c, $\dagger$ respectively). (b) Capacitance retention with increasing current density. (c) Cycling stability evaluated at a high current density of $10 \mathrm{~A} \mathrm{~g}^{-1}$. (d) Nyquist plots under open-circuit voltage.

$\left(C_{\mathrm{s}}\right)$ of BNCHPs was calculated to be $385 \mathrm{~F} \mathrm{~g}^{-1}$ at $0.1 \mathrm{~A} \mathrm{~g}^{-1}$ (Fig. 6b), according to the formula: $C_{\mathrm{s}}=I \Delta t / \Delta V m$, where $I$ denotes the current; $\Delta t$ is the discharge time; $\Delta V$ represents the voltage change during the discharge; and $m$ expresses the total mass of the active materials in the electrode. ${ }^{25}$ Such a capacitance is superior to that $\left(\sim 305 \mathrm{~F} \mathrm{~g} \mathrm{~g}^{-1}\right.$ at $\left.0.1 \mathrm{~A} \mathrm{~g}^{-1}\right)$ of the spherical counterpart, i.e. NCHSs with structural and chemical characteristics similar to those of BNCHPs (including size, surface area, and nitrogen content, etc., see Fig. S4 and Table S1†), which were prepared using PS hollow spheres as the template under similar experimental conditions. The specific capacitance of BNCHPs is also better than those of many reported porous carbon materials with larger surface areas, such as $\mathrm{N}$-doped porous carbons as well as graphenebased porous carbon materials (Table S2 $\dagger$ ). ${ }^{17-21}$ In addition, the volumetric density $\left(\rho_{\mathrm{v}}\right)$ of BNCHPs was measured to be $\sim 1.07 \mathrm{~g} \mathrm{~cm}^{-3}$, which is much larger than that $\left(\sim 0.64 \mathrm{~g} \mathrm{~cm}^{-3}\right)$ of NCHSs (the estimation of the volumetric density followed a reported method ${ }^{25,26}$ and the details are given in Table $\mathrm{S} 1 \dagger$ ). Accordingly, the volumetric capacitance $\left(C_{\mathrm{v}}=C_{\mathrm{S}} \times \rho_{\mathrm{v}}\right)$ of BNCHPs is estimated to be $\sim 410 \mathrm{~F} \mathrm{~cm}^{-3}$ at $0.1 \mathrm{~A} \mathrm{~g}^{-1}$, greater than $\sim 190 \mathrm{~F} \mathrm{~cm}^{-3}$ for NCHSs, as well as those of many reported carbon materials (Table $\mathrm{S} 3 \dagger){ }^{22-26}$

BNCHPs also exhibited good capacitance retention (Fig. 6b). A high specific capacitance of $\sim 200 \mathrm{~F} \mathrm{~g}^{-1}$ was attained at a current density of $2.0 \mathrm{~A} \mathrm{~g}^{-1}$, and this value remained stable with increasing the current density. The capacitance reduction at current densities higher than 0.1 $\mathrm{A}^{-1}$ is owing to the ion transport limitation, which often occurs in electrode materials at high current densities. ${ }^{19}$ It is well-known that the rate capability of electrode materials is mainly determined by the kinetics of ion diffusion and 
electronic conductivity. At lower current densities, the ions can sufficiently diffuse into almost all holes of the electrode while the effective contact between the ions and the electrode is greatly reduced at higher current densities, leading to a lower capacitance. On the other hand, the cycling stability of BNCHPs was evaluated by galvanostatic charge/discharge measurements at a high current density of $10 \mathrm{~A} \mathrm{~g}^{-1}$. Remarkably, almost no capacitance loss was observed after 10000 cycles, indicating an excellent cycling stability of BNCHPs as electrode materials (Fig. 6c).

To further understand the superior capacitor performance of BNCHPs, electrochemical impedance spectra of BNCHPs and NCHSs were recorded (Fig. 6d). These spectra do not show obvious semicircle regions, probably due to the low resistances of BNCHPs and NCHSs. ${ }^{33}$ In addition, the spectra indicate that a smaller resistance $(\sim 1.3 \Omega)$ of BNCHPs than that $(\sim 1.7 \Omega)$ of NCHSs. This is probably because the bowl-like particles have more contact area with neighbouring particles compared with spherical ones, which enhances electron transport. The lower resistance plays a crucial role in enhancing the electrical conductivity and power output of supercapacitors. ${ }^{34}$

The superior electrochemical performance of BNCHPs can be attributed to their unique bowl-shaped structure along with hierarchically porous architecture which provides a synergistic effect of macro- and meso-/micropores, as illustrated in Fig. 2b. First, the macropores in the bowl-shaped hollow particles and derived from their stacking can reserve electrolytes thus shorten their diffusion distances from the external to the internal surfaces; the meso-/micropores as well as the suitable nitrogen doping in the carbon shells increase specific surface area, enhance ion transport, and improve the efficiency of charge storage. Second, the smaller cavities of the bowl-shaped hollow particles in comparison with those of their spherical counterpart shorten the diffusion distances of electrolytes to the carbon shells. In addition, the bowl-like particles have more contact area with neighbouring particles compared with spherical ones, which enhances charge transport and structural stability of electrodes. Third, the bowl-like particles largely decrease their volumes by reducing the empty cavity in comparison with their spherical counterpart, and thus increase the packing density and consequently the volumetric capacitance of active materials.

\section{Conclusions}

In summary, we developed a new potential application for BCP vesicles in supercapacitors, as well as a facile self-assembly strategy towards bowl-shaped carbon-containing hollow particles using kippah vesicles as the template. The resulting bowl-shaped materials, BNCHPs, show superior electrochemical performance as electrode materials of supercapacitors, with high specific capacitance of up to $385 \mathrm{~F} \mathrm{~g}^{-1}$ at $0.1 \mathrm{~A} \mathrm{~g}^{-1}$ (corresponding to a high volumetric capacitance of $\sim 410$ $\mathrm{F} \mathrm{cm}^{-3}$ ), good capacitance retention, and excellent cycling stability with no capacitance loss after 10000 cycles. This study took advantage of block copolymer self-assembly, a versatile strategy in morphological control of superstructures, for the fabrication of bowl-shaped carbon materials for high performance supercapacitors. It can be expected that this strategy may help to expand the family of bowl-shaped materials, by developing carbon-metal, carbon-metal oxide hybrids, and many other types of nanomaterials, for energy-related applications.

\section{Acknowledgements}

The authors appreciate the financial support from 973 Programs of China (2012CB933404 and 2013CBA01602), Natural Science Foundation of China (21320102006, 21304057 and 51573091), Natural Science Foundation of Shanghai (13ZR1421200), Program for Eastern Scholar in Shanghai, Ph.D. Programs of Foundation of Ministry of Education of China for Young Scholars (20130073120088). We also thank the Instrumental Analysis Center of Shanghai Jiao Tong University for some measurements.

\section{Notes and references}

1 A. Blanazs, S. P. Armes and A. J. Ryan, Macromol. Rapid Commun., 2009, 30, 267-277.

2 Y. Mai and A. Eisenberg, Chem. Soc. Rev., 2012, 41, 59695985.

3 K. Yu, C. Bartels and A. Eisenberg, Langmuir, 1999, 15, 7157-7167.

4 D. E. Discher and A. Eisenberg, Science, 2002, 297, 967-973.

5 R. Zheng and G. Liu, Macromolecules, 2007, 40, 5116-5121.

6 K. T. Kim, J. Zhu, S. A. Meeuwissen, J. J. L. M. Cornelissen, D. J. Pochan, R. J. M. Nolte and J. C. M. van Hest, J. Am. Chem. Soc., 2010, 132, 12522-12524.

7 R. J. Hickey, A. S. Haynes, J. M. Kikkawa and S.-J. Park, J. Am. Chem. Soc., 2011, 133, 1517-1525.

8 J. Qin, Q. Liu, J. Zhang, J. Chen, S. Chen, Y. Zhao and J. Du, ACS Appl. Mater. Interfaces, 2015, 7, 14043-14052.

9 L. Wang, G. Liu, X. Wang, J. Hu, G. Zhang and S. Liu, Macromolecules, 2015, 48, 7262-7272.

10 T. Azzam and A. Eisenberg, Langmuir, 2010, 26, 1051310523. "Kippah" means a dome or a traditional skullcap.

11 S. Yang, X. Feng, L. Zhi, Q. Cao, J. Maier and K. Müllen, Adv. Mater., 2010, 22, 838-842.

12 S. Ding, J. Chen, G. Qi, X. Duan, Z. Wang, E. Giannelis, L. A. Archer and X. W. Lou, J. Am. Chem. Soc., 2011, 133, 21-23.

13 X. Lai, J. E. Halpert and D. Wang, Energy Environ. Sci., 2012, 5, 5604-5618.

14 Z. Qiao, B. Guo, A. J. Binder, J. Chen, G. M. Veith and S. Dai, Nano Lett., 2013, 13, 207-212.

15 F. Xu, Z. Tang, S. Huang, L. Chen, Y. Liang, W. Mai, H. Zhong, R. Fu and D. Wu, Nat. Commun., 2015, 6, 7221. 
16 J. Li, K. Liu, X. Gao, B. Yao, K. Huo, Y. Cheng, X. Cheng, D. Chen, B. Wang, W. Sun, D. Ding, M. Liu and L. Huang, ACS Appl. Mater. Interfaces, 2015, 7, 24622-24628.

17 H. Wang, Q. Gao and J. Hu, Microporous Mesoporous Mater., 2010, 131, 89-96.

18 L. Zhang and G. Shi, J. Phys. Chem. C, 2011, 115, 1720617212.

19 Z. Wu, Y. Sun, Y. Tan, S. Yang, X. Feng and K. Müllen, J. Am. Chem. Soc., 2012, 134, 19532-19535.

20 P. Chen, J. Yang, S. Li, Z. Wang, T. Xiao, Y. Qian and S. Yu, Nano Energy, 2013, 2, 249-256.

21 J. H. Lee, N. Park, B. G. Kim, D. S. Jung, K. Im, J. Hur and J. W. Choi, ACS Nano, 2013, 7, 9366-9374.

22 D. Hulicova-Jurcakova, M. Kodama, S. Shiraishi, H. Hatori, Z. Zhu and G. Lu, Adv. Funct. Mater., 2009, 19, 18001809.

23 M. Heon, S. Lofland, J. Applegate, R. Nolte, E. Cortes, J. Hettinger, D. P.-L. Taberna, P. Simon, P. Huang, M. Brunet and Y. Gogotsi, Energy Environ. Sci., 2011, 4, 135-138.

24 Z. Lei, L. Lu and X. Zhao, Energy Environ. Sci., 2012, 5, 6391-6399.
25 M. Ghaffari, Y. Zhou, H. Xu, M. Lin, T. Y. Kim, R. S. Ruoff and Q. Zhang, Adv. Mater., 2013, 25, 4879-4885.

26 Y. Tao, X. Xie, W. Lv, D. Tang, D. Kong, Z. Huang, H. Nishihara, T. Ishii, B. Li, D. Golberg, F. Kang, T. Kyotani and Q. Yang, Sci. Rep., 2013, 3, 2975-2982.

27 Y. Mai and A. Eisenberg, J. Am. Chem. Soc., 2010, 132, 10078-10084.

28 K.-C. Kao, C.-J. Tsou and C.-Y. Mou, Chem. Commun., 2012, 48, 3454-3456.

29 J. Liang, H. Hu, H. Park, C. Xiao, S. Ding, U. Paik and X. W. Lou, Energy Environ. Sci., 2015, 8, 1707-1711.

30 D. Liu, X. Peng, B. Wu, X. Zheng, T. T. Chuong, J. Li, S. Sun and G. D. Stucky, J. Am. Chem. Soc., 2015, 137, 9772-9775.

31 U. H. F. Bunz, K. Seehafer, F. L. Geyer, M. Bender, I. Braun, E. Smarsly and J. Macromol Freudenberg, Macromol. Rapid Commun., 2014, 35, 1466-1496.

32 X. Zhuang, F. Zhang, D. Wu, N. Forler, H. Liang, M. Wagner, D. Gehrig, M. R. Hansen, F. Laquai and X. Feng, Angew. Chem., Int. Ed., 2013, 52, 9850-9854.

33 J. Bae, M. K. Song, Y. J. Park, J. M. Kim, M. Liu and Z. Wang, Angew. Chem., Int. Ed., 2011, 50, 1683-1687.

34 L. Hao, X. Li and L. Zhi, Adv. Mater., 2013, 25, 3899-3904. 